ISSN: 2539-3804@) @®)

\title{
Transferencia y Contraste. Una metodología semiológica para el aprendizaje del francés por hispanohablantes
}

Claudia Lucia Mejía Quijano

Daniel Jaramillo Giraldo

José Luis Betancur

Universidad de Antioquia

Medellín, Colombia

\section{Resumen}

En este artículo metodológico mencionaremos desde nuestra óptica semiológica, primero, los problemas pedagógicos que hemos encontrado de manera recurrente en la docencia del francés en un programa de traducción francés-español. Luego, esbozaremos las bases semiológicas que nos han permitido vislumbrar algunas soluciones para estos problemas, y terminaremos exponiendo una propuesta metodológica, que sistematizamos en un curso, así como lo observado en esta implementación y las perspectivas a las que dio lugar, lo que analizaremos desde un punto de vista lingüístico.

Palabras clave: didáctica; semiología; transferencia; comparación; escritura; AFI.

\begin{abstract}
Transfer and Contrast. A semiological methodology for learning French by Spanish speakers

This methodological article will first discuss, from a semiological point of view, the pedagogical problems that we have encountered in the teaching of French in a FrenchSpanish translation program. Then, the semiological bases that allowed to consider some solutions for these problems will be outlined, and it will end by presenting a methodological proposal, which was systematized in a course, as well as the observations made during this implementation and the perspectives to which it gave rise, that will be analyzed from a linguistic point of view.
\end{abstract}

Key words: didactics; semiology; transfer; comparison; writing; IPA. 


\section{Résumé}

Transfert et Contraste. Une méthodologie sémiologique pour l'apprentissage du français par des hispanophones

Dans cet article méthodologique, à partir de notre optique sémiologique, on abordera d'abord les problèmes pédagogiques trouvés de façon récurrente dans l'enseignement du français dans un programme de traduction français-espagnol. Ensuite, on esquissera les fondements sémiologiques qui ont permis d'en envisager quelques solutions, et on finira par l'exposé d'une proposition méthodologique, que nous avons mise à l'épreuve dans un cours, ainsi que les observations réalisées et les perspectives qui en ont résulté, ce que nous analyserons d'un point de vue linguistique.

Mots-clés : didactique ; sémiologie ; transfert ; comparaison ; écriture ; API. 


\section{CÓMO CITAR ESTE ARTÍCULO}

Mejía, C., Jaramillo, D., \& Betancur, J. (2020). Transferencia y Contraste. Una metodología semiológica para el aprendizaje del francés por hispanohablantes. Lenguaje, 48(1), 84-123. doi: 10.25100/lenguaje.v48i1.8575 


\section{INTRODUCCIÓN}

Aprender una lengua quiere decir ante todo poder comunicarse en esa lengua, poder comprenderla y hablarla. Ahora bien, fuera del contexto francohablante, es corriente que los estudiantes hispanohablantes aprendan mejor a leer y escribir el francés, como lo señala Bartolí (2005) para su región catalana: “Aunque los alumnos desean destacar en las destrezas orales, en general, llegan a tener conocimientos superiores sólo en la lengua escrita" (p. 2). En general, todo hablante alfabetizado ya presenta este desfase en su primera lengua: hay quienes hablan mejor de lo que escriben, y otros escriben mejor de lo que hablan, pues las destrezas que requieren las lenguas orales y las lenguas escritas son bastante diferentes. No es de extrañar que este desfase primario aumente en las segundas lenguas, generalmente según la práctica que se le dé a la nueva lengua, pero también según el contraste entre la estructura lingüística de la primera y la de la segunda lengua. En particular, las lenguas orales implican nuevos hábitos articulatorios, difíciles de adoptar cuando los maternos son muy distintos, pues estos últimos están bien arraigados y se han vuelto casi reflejos articulatorios. Tan es así que todavía hay quien afirme que no es realista que los adultos aprendan a hablar como un nativo una lengua extranjera como el inglés: “(...) evidence for the critical period hypothesis, suggesting that native-like pronunciation was an unrealistic goal for older second language learners, led to the argument that instructional time would be better spent on teaching something that learners could learn more successfully" (Lightbown y Spada, 2013, p. 68).

Este tipo de afirmaciones se ha repetido desde hace mucho y corresponde en parte a la experiencia común de los acentos regionales, difíciles de cambiar en la lengua materna por la relación entre el acento y la identidad de los hablantes, pero extrapolar a las lenguas segundas este aspecto puede dar lugar a prejuicios, pues el estudio científico de los sonidos, la fonética instrumental, ha demostrado que, aunque es difícil, no es algo imposible (Suso, 2002).

En nuestro contexto, también hemos observado en los estudiantes este desfase entre lo oral y lo escrito, pero lo específico del programa de traducción al que pertenecemos nos ha hecho ver también otros aspectos. Para los hispanohablantes que aprenden el francés las dificultades orales no sólo son cuantitativamente considerables, sino que se pueden convertir en una diferencia cualitativa consecuente, en particular porque el francés oral está muy alejado de su escritura y la ortografía de las palabras francesas no corresponde a su forma oral, lo que para los mismos francohablantes es un grave problema, que suscita regularmente intentos de reformas ortográficas y nutridos debates en toda la sociedad francesa desde hace varios siglos.

Recordemos que el francés y el español comparten un mismo origen latino y por esto muchas palabras escritas de ambas lenguas se parecen entre sí, a pesar de que tengan valores semánticos y sintácticos diferentes; es decir que hay más falsos cognados entre el 
español escrito y el francés escrito que entre las lenguas orales respectivas, las cuales han evolucionado en forma divergente y se han diferenciado de manera casi absoluta. Es muy probable que esta similitud escrita influya positivamente en las destrezas escritas mencionadas por Bartolí (2005), ya que para un hispanohablante leer el francés es aparentemente más fácil que oírlo.

Sin embargo, en nuestra experiencia en un programa de traducción, hemos observado que esta semejanza únicamente escrita también posee un aspecto negativo, pues lleva a los estudiantes a realizar correspondencias engañosas entre las palabras y entre la sintaxis de ambas lenguas. En el nivel semántico, en particular, la semejanza da lugar a innumerables calcos, difíciles de ver para los mismos estudiantes, quienes sin darse cuenta atribuyen sentidos "españoles" a las nuevas palabras francesas que ven escritas. Estos calcos semánticos son tan importantes que se podría considerar que, cuando leen, sobre todo al principio del aprendizaje, los estudiantes entienden el francés con los valores sintácticos y semánticos del español, y, cuando tratan de escribir con palabras francesas, lo hacen con los valores sintácticos y semánticos del español, y esto ocurre -aquí radica el mayor problema- sin que se den cuenta de la mezcla que realizan. En el transcurso del aprendizaje de una segunda lengua, es normal que se cree en un momento dado una especie de "interlingua" que permite avanzar al estudiante. Sin embargo, en el caso del francés y del español, esta mezcla puede perjudicar el aprendizaje.

En efecto, cuando la interferencia del español en el aprendizaje del francés es muy importante, se puede llegar a crear en la mente de los estudiantes una verdadera simbiosis implícita entre ambas lenguas, simbiosis que varía en magnitud según cada estudiante, y que sólo va disminuyendo según el contacto que este logre tener con el francés oral, pues el francés escrito sólo alimenta la simbiosis. Hemos podido observar fácilmente esta simbiosis en nuestra enseñanza enfocada al aprendizaje de la lengua con fines traductivos, pues en nuestro programa se le da lógicamente gran importancia a la lengua escrita en el transcurso del aprendizaje, pero, para poder traducir, es asimismo muy importante que los valores de las lenguas en contacto estén bien diferenciados.

Hay que resaltar que nuestra universidad es pública y recibe estudiantes sin ningún conocimiento previo del francés: en el primer semestre los estudiantes comienzan a aprender la lengua desde el nivel de debutante absoluto, y en dos años de ciclo básico deben dominar lo necesario de la lengua para luego seguir dos años de traducción profesional. Los intercambios a países francohablantes, que pueden realizar después del ciclo básico, les permiten corregir muchos calcos y al final de los estudios, para nuestra siempre sorprendida satisfacción, muchos estudiantes tienen un buen desempeño. Sin embargo, algunos estudiantes no realizan el intercambio o escogen intercambios en países anglohablantes, lo que les dificulta luego la parte francesa del ciclo profesional. Son las falencias recurrentes de estos estudiantes las que nos han invitado a reflexionar sobre la manera de enseñar el francés en nuestro programa. 
Como también sucede en otros ámbitos didácticos diferentes, es común que la enseñanza del francés empiece con la explicación de los diferentes fonemas de la lengua: los estudiantes realizan ejercicios de reconocimiento y tratan de articular los fonemas, pero como toda la lengua debe ser adquirida en dos años, de tal manera que luego puedan leer los textos que aprenderán a traducir, en general no se pasa mucho tiempo en esta parte fonológica, sino que rápidamente los estudiantes leen textos y se les pide que entiendan frases enteras, con palabras que por lo general han oído poco, aunque sí las hayan visto escritas y aprendido en los libros de estudio. Es decir que, antes de que se les haya dejado el tiempo para que asimilen el universo oral del francés; o sea antes de que logren oír perfectamente los fonemas, los grupos fónicos, la entonación, la prosodia, la sintaxis fonológica del francés; antes de que dominen nuevos hábitos articulatorios y puedan articular con claridad las cadenas fónicas francesas con su respectiva prosodia, elementos que son todos bastante alejados de los de la lengua materna, los estudiantes deben entender y producir la lengua mediante un aprendizaje que se da esencialmente por escrito.

Cuando este aprendizaje mayoritariamente escrito se imparte en un medio francohablante, en el que el estudiante tiene la oportunidad de oír y articular la lengua en suficientes ocasiones, no existe mayor inconveniente, pues la experiencia oral constante complementa el aprendizaje escrito y corrige la falsa impresión de comprensión a partir del español (los intercambios estudiantiles también cumplen esta función). Sin embargo, cuando se trata de una enseñanza fuera del contexto francohablante, como es nuestro caso, lo escrito se vuelve demasiado preponderante dando lugar a la posibilidad de mezclar ambas lenguas, mezcla palpable cuando se les pide a los estudiantes que por primera vez traduzcan un texto del francés al español. Si en la didáctica general de una segunda lengua esta mezcla puede no presentar dificultades a los docentes, para la didáctica con el fin específico de la traducción, la simbiosis que se crea es contraproducente y no permite que se instaure rápidamente un proceso traductivo adecuado.

En efecto, la traducción es una actividad que puede servir de lupa, permitiendo ver el conocimiento idiomático del traductor con respecto a la lengua meta, pero también con respecto a la lengua del texto que se quiere traducir: en general, nuestros estudiantes escriben relativamente bien en su lengua materna, pero, al traducir, pueden producir muchas frases "cojas" en español, y cuando estos deslices no corresponden a sus competencias en redacción española, provienen generalmente de la contaminación del francés, pero también de la mezcla previa que han construido al aprender esta lengua. Puede ocurrir que durante el aprendizaje algunos estudiantes lleguen a instaurar verdaderas equivalencias erróneas entre las dos lenguas, provocando malentendidos que los descorazonan e instauran un círculo vicioso entre impotencia y desagrado por la lengua: la mezcla de lenguas implica no sólo que a los estudiantes se les dificulta escuchar 
(lo que crea temores frente a sus propias posibilidades, y lo que a su vez frena el aprendizaje) y experimentan bloqueos cuando quieren hablar francés (lo que los frustra), sino también una falta de comprensión importante en el nivel que se requiere para traducir, el cual es más profundo que una comprensión global de los actos comunicativos y se basa de manera importante en el texto: al leer, los estudiantes confunden el sentido de las frases escritas, y por consiguiente presentan esa producción pobre en las traducciones al español.

Paradójicamente, fue entonces en la enseñanza del francés con fines traductivos donde surgió la inquietud por darle un lugar preponderante al francés oral en el aprendizaje temprano de la lengua. Tal vez, no sería de extrañar que uno de los factores que influyan en el desfase en los estudiantes entre las competencias orales y escritas del francés sea esta misma preponderancia de lo escrito en el aprendizaje temprano de la lengua y el poco tiempo que se le dedica al aprendizaje de las bases orales del francés, no sólo en nuestro programa de traducción, sino también en otros contextos didácticos.

\section{Francés oral y francés escrito}

En general, muchos autores han mencionado la dificultad del aprendizaje del francés oral (Bartolí, 2005; Bolekia, 1989; Detey, 2007; Giralt, 2006; Guimbretière, 2000) y todos están de acuerdo en que es indispensable mejorar las competencias orales de quienes aprenden francés. Podemos citar un ejemplo histórico: en el siglo XIX, Francisco Piferrer (1847) ya proponía un método particular con pintorescas notaciones fonéticas inventadas por él mismo, que incluía un trabajo considerable sobre lo que llamaban en la época la pronunciación del francés.

Para explicar nuestra posición a este respecto, tenemos que precisar los términos que vamos a emplear. En este artículo no utilizaremos las nociones de pronunciación, corrección fonética, ni la noción más fisiológica de fonética del francés, que corresponden a puntos de vista ajenos al punto de vista semiológico. Cuando se habla de pronunciación, se tiene como foco más importante las palabras escritas que se consideran la verdadera lengua, como lo señala Ferdinand de Saussure (2019) en su primer curso de lingüística general:

Tendemos a olvidar que aprendimos a hablar antes de saber escribir y, luego, invertimos la relación. El mejor indicio de esta concepción es la manera como se entiende la pronunciación de una palabra. Frente a una palabra escrita uno se pregunta cómo hay que pronunciarla... ¡cual si fuera la ejecución de un signo escrito, como en la música...! O sea que se parte de la palabra escrita, cuando en realidad es imposible tomarla como base de nuestro estudio. (p. 103). 
En cuanto a la corrección fonética, esta es una expresión que se utiliza en contextos normativos y la fonética del francés implica un estudio lingüístico profundo que no corresponde al campo de la enseñanza de lenguas con fines prácticos.

Utilizaremos en cambio las nociones de francés escrito y francés oral desde una perspectiva semiológica. Sin embargo, existe una homonimia con estas últimas expresiones que tenemos que aclarar, pues estas nociones en semiología saussureana no son las que han sido utilizadas, con las mismas palabras, en los trabajos de lingüistas que desarrollaron en el siglo XX el estudio de lo oral y las relaciones con lo escrito. Por ejemplo, los trabajos descriptivos de Claire Blanche-Benveniste y de su equipo, que tienen gran valor para la lengua francesa, pero que no tomamos como referencia, porque su óptica es únicamente lingüística; para ella lo oral y lo escrito son dos aspectos de una sola lengua, aspectos que no son absolutamente idénticos, pero que tampoco son esencialmente diferentes, según se afirma en varios textos, como este:

Les études menées sur de grands corpus de français parlé font découvrir des mécanismes linguistiques généraux, qui concernent aussi bien le versant oral que le versant écrit de la langue ; elles ne révèlent pas une nature spéciale de systèmes linguistiques qui seraient propres à l'oral et à l'écrit. (Blanche-Benveniste, 1991, p. $52)$.

El valor de los trabajos de Blanche-Benveniste fue sacar lo oral del desprestigio en el que estaba frente a lo escrito; por ejemplo, debatiendo, en relación con la evolución de las lenguas, los a prioris de Henri Frei en su Grammaire des fautes, y mostrando lo reductor del punto de vista normativo: "Sous le terme de « français parlé » [de Frei, entre otros], il conviendra toujours de comprendre, de façon très restrictive, le français "fautif », et particulièrement dans le domaine de la syntaxe" (Blanche-Benveniste, 1995, p. 26).

Esta postura contra la sobrevaloración de lo escrito implica que para BlancheBenveniste el francés escrito y el francés oral no son sino dos aspectos de una sola lengua francesa, y en su obra trató de encontrar el mismo funcionamiento profundo entre ambos aspectos, limitando las divergencias al material, fónico o visual en cada caso. Al contrario, desde la semiología saussureana, vamos a distinguir el francés oral y el francés escrito como dos sistemas semiológicos que, aunque mantienen vínculos estrechos y se corresponden en ciertos aspectos, no son en absoluto equivalentes y pueden constituir por lo tanto dos entidades independientes. En este apartado mostraremos esa diferencia semiológica, aunque, por el marco metodológico de este artículo, sin entrar de lleno en el debate teórico y limitándonos a la discusión práctica sobre la enseñanza del francés. 


\section{El primer paso}

Sin embargo, antes de entrar en esa explicación, precisemos que, a nivel de la enseñanza de la lengua, aunque todos los autores concuerden en que la enseñanza del francés oral debe mejorarse, aunque sea preferible que el francés oral se enseñe antes que el francés escrito (Detey, 2007), aunque nadie niegue que el núcleo de la enseñanza de la lengua deben ser las competencias comunicativas, principalmente las orales, hay que observar que poco de esto ha llegado realmente al salón de clase. En particular, como los hispanohablantes fácilmente creen entender el francés escrito, es raro que la escritura no se tome en cuenta en las primeras fases del aprendizaje de la lengua, aunque lo contrario sería algo bastante obvio: al principio se debería aprender más bien a escuchar y a hablar, es decir que sólo después de tener bases firmes del francés oral, se debería comenzar el aprendizaje del francés escrito propiamente dicho: es así como los niños aprenden naturalmente su lengua materna.

Precisemos, sin embargo, que no nos referimos aquí al ya conocido método audiolingual o verbo-tonal (Alcalde, 2011; Richards y Rogers, 1986), pues se trata de aprender una lengua oral y no únicamente de adquirir hábitos articulatorios y saber pronunciar bien el francés: este fue el objetivo de los métodos que nacieron en el siglo XX y que se basaron en los avances de la fonética y la fonología (Suso, 2002), métodos que, por la óptica normativa que tomaban en su mayoría, dejaron de lado el componente comunicativo de las lenguas, lo que se les criticó fuertemente. Tampoco se trata, desde luego, de enseñar el francés tal como los niños aprenden su lengua materna, lo que sería imposible, pues los adultos ya tienen una lengua que es la base innegable de cualquier aprendizaje ulterior. Sólo proponemos una pauta metodológica: distinguir la lengua oral de la lengua escrita y empezar el aprendizaje únicamente por la lengua oral, para luego, cuando se introduzca el francés escrito, poder establecer los vínculos necesarios entre ambas lenguas, como ocurre en el aprendizaje normal de la lengua materna.

Cuando examinamos esta propuesta, la primera pregunta que se plantea es:

¿es teóricamente posible enseñar y aprender una lengua oral de manera exclusiva, es decir sin la lengua escrita correspondiente?

Si pensamos en las lenguas maternas, la respuesta es obvia: la lengua oral sí se puede aprender y estudiar con total independencia de la lengua escrita, como lo muestran además las múltiples lenguas que han existido sin haber sido escritas. Estas lenguas únicamente orales permitieron incluso el desarrollo de literaturas: Homero se transmitió oralmente durante mucho tiempo antes de que se fijara por escrito, los grandes poemas épicos del origen fueron transmitidos primero oralmente. También se puede pensar en las venerables tradiciones africanas $\mathrm{y}$, más cerca de nosotros, en nuestras lenguas indígenas, pero, sobre todo, cada niño siempre aprende su lengua materna sólo oralmente durante varios años, antes de comenzar a aprender la lengua escrita, si esta existe. 
Si teóricamente es posible aprender una lengua oral sin ayuda de la lengua escrita correspondiente, entonces ¿por qué no es común hacer esto con las segundas lenguas?

La primera razón que explicaría esta situación es la dificultad práctica de enseñar relativamente rápido una segunda lengua oral sin ayuda de la lengua escrita correspondiente. En lo referente al trabajo didáctico con grupos numerosos, está claro que un docente no puede dedicar el tiempo necesario a una adecuada reorganización de las destrezas orales, pues esto implicaría un exigente trabajo individual con cada estudiante, mientras que los ejercicios escritos en papel se pueden distribuir y corregir de manera más ágil y al mismo tiempo a varios estudiantes.

Además de esta razón de comodidad para el docente, también se puede argumentar que, para los estudiantes que ya dominan su lengua materna escrita, aprender de manera únicamente oral es más lento que aprender con ayuda de la escritura, debido a la naturaleza efímera de los sonidos: las palabras desaparecen una vez pronunciadas, por lo que exigen un trabajo de memoria mayor, que depende tanto de las cualidades sensoriales de cada quien como de las prácticas individuales ejercidas. Por ejemplo, un ciego o un músico desarrollan una memoria acústica impresionante (y aún más un músico ciego, como Ray Charles); los sordos y los pintores, por su parte, tienen capacidades motrices y visuales muy superiores. La memoria únicamente acústica no está muy desarrollada en la mayoría de las personas, sobre todo en las comunidades alfabetizadas. Es por esto que los que ven y oyen, que constituyen la mayor parte de los estudiantes de francés, memorizan mejor si se combina lo acústico con lo visual.

Por lo tanto, en la práctica, tanto para el docente como para el estudiante, las desventajas de un aprendizaje únicamente oral son reales.

También se podría argumentar teóricamente el gran valor que tiene aprender la lengua escrita. En nuestro caso de una enseñanza para traductores, tenemos que introducir el francés escrito rápidamente. Ahora bien, sin negar este valor de la escritura y la gran autoridad que tiene, ni la necesidad de enseñar el francés escrito, podemos tener en cuenta que la lengua oral no solamente es autónoma, sino que, para los semiólogos saussureanos, es la que es "la lengua", pues la escritura es otro tipo de sistema semiológico, que puede ser autónomo.

En este momento de la argumentación, es necesario introducir varios conceptos semiológicos que nos parecen útiles para elucidar nuestro problema y aclarar nuestra posición. Saussure propuso la Semiología como la disciplina que estudia los diferentes sistemas que las comunidades humanas han creado para comunicarse, y lo expresa así en su tercer curso de lingüística general (Constantin y Saussure, 2005):

Avant d'aller plus loin, il faut interposer une autre idée: c'est celle des faits sémiologiques dans les sociétés. Reprenons la langue considérée comme un 
produit du travail social : c'est un ensemble de signes fixés par un accord des membres de cette société; ces signes évoquent des idées, mais par les signes ça a quelque chose de commun avec les rites par exemple. Presque toutes les institutions, pourrait-on dire, ont à la base des signes, mais ils n'évoquent pas directement les choses. Il se passe dans toutes les sociétés ce phénomène que pour différents buts des systèmes de signes évoquant directement les idées que l'on veut se trouvent établis ; il est évident que la langue est un de ces systèmes et que c'est de tous le plus important, mais il n'est pas l'unique, et par conséquent nous ne pouvons pas laisser de côté les autres. Il faudrait donc faire entrer la langue dans les institutions sémiologiques : celle des signaux maritimes par exemple (signes visuels), les signaux de trompette militaires, le langage par signes des sourdsmuets, etc. L'écriture est également un vaste système de signes. Il y aura une psychologie des systèmes de signes, cette psychologie sera une partie de la psychologie sociale, c'est-à-dire ne sera que sociale; il s'agira de la même psychologie qui est applicable à la langue. Les lois de transformations de ces systèmes de signes auront souvent des analogies tout à fait topiques avec les lois de transformations de la langue. C'est une observation facile à faire pour l'écriture, - quoique ce soient des signes visuels - qui subit des altérations comparables à des phénomènes phonétiques. (p. 89).

Desde las primeras reflexiones sobre el signo a nivel general, que encontramos en manuscritos de 1894, Saussure (1968) tuvo en cuenta la escritura al lado del signo lingüístico, para conformar el campo semiológico: “Le langage (...) c'est une institution humaine, mais de telle nature que toutes les autres institutions humaines, sauf celle de l'écriture, ne peuvent que nous tromper sur sa véritable essence [...] le langage et l'écriture ne sont PAS FONDÉS sur un rapport naturel des choses" (p. 169). Sin embargo, estos dos sistemas, aunque sean en realidad independientes, se han mezclado siempre por su misma naturaleza semiológica, creando muchos malentendidos. Saussure (2019) ataca fuertemente esta mezcla en el primer curso de lingüística general: "La confusión entre la lengua y la escritura es nefasta para la lingüística" (p. 95), "una lengua es completamente independiente de la escritura" (p. 105).

En la estela de lo trabajado por Saussure, la escritura fue un objeto de estudio predilecto para los semiólogos de orientación saussureana, como el argentino Luis Prieto, quien, en su artículo Lingue grafiche e lingue foniche (1983), desarrollando lo expresado en un artículo anterior de Saussure (1974), propuso distinguir los alfabetos como códigos sustitutivos y concebir los sistemas gráficos como verdaderas lenguas escritas:

les systèmes formés par les signes graphiques satisfont toutes les conditions pour pouvoir considérer qu'ils constituent des langues, l'emploi de ce terme doit être étendu à ces systèmes, quitte à spécifier, chaque fois que cela s'avère nécessaire, s'il 
s'agit d'une 'langue phonique' ou d'une 'langue graphique'. (Prieto, 1983, p. 9, trad. revisada por el autor).

Consideramos, con estos autores, que la escritura puede constituir un sistema semiológico independiente como las lenguas orales, aunque los alfabetos de estas lenguas constituyen asimismo códigos sustitutivos. Para el propósito pedagógico, proponemos entonces que se tenga en cuenta esta naturaleza dual de la escritura.

La escritura se creó al principio de la historia como un simple código sustitutivo de la forma sonora de los signos, es decir se crearon dibujos como equivalentes visuales de las palabras-sílabas (escrituras ideográficas), y luego letras como equivalentes de los fonemas de la lengua oral (escrituras alfabéticas), pero el uso de este código sustitutivo fue convirtiéndolo, con el desarrollo de las diferentes civilizaciones humanas, en un sistema semiológico autónomo que transmite tradiciones culturales. Actualmente, nuestras escrituras poseen esta naturaleza dual que proviene de su historia, y funcionan de manera distinta según si se observa el código sustitutivo o el sistema autónomo.

\section{La escritura: código sustitutivo y lengua}

Cuando distinguimos estos dos aspectos de la escritura podemos darnos cuenta de que el que se utiliza en el aprendizaje temprano del francés es el código sustitutivo, es decir se aprenden las letras que simbolizan los fonemas del francés y los conjuntos de letras que corresponden a palabras del francés. Por su parte, el aspecto de la escritura que se utiliza al final del aprendizaje es el sistema autónomo, que sigue reglas complejas de composición (sintaxis, puntuación, anafóricos, conectores específicos, etc.).

La escritura francesa está muy desarrollada como sistema semiológico y los mismos francohablantes deben realizar estudios prolongados para llegar a dominar esta verdadera lengua escrita, pues tiene una tradición de varios siglos, que es estricta, normativa y monolítica, y en numerosos aspectos no concuerda con la lengua oral ${ }^{1}$. En particular, este sistema marca las diferencias de género y número de sujetos, y en ciertos casos de complementos directos, en todas las palabras de los sintagmas, lo que lo diferencia del francés oral que no marca el género y el número en todas las palabras, sino sólo en determinadas partes de los sintagmas. El francés escrito, como sistema semiológico, también marca las terminaciones de las conjugaciones verbales cuando el francés oral las ha ido perdiendo y tiene cada vez más un sistema verbal divergente del sistema verbal del francés escrito, el cual hasta posee varios tiempos desaparecidos en el francés oral (pasado simple y los tiempos del subjuntivo).

\footnotetext{
${ }^{1}$ Cabe señalar que estas afirmaciones concuerdan en parte con lo observado en la enseñanza del francés en otros contextos lingüísticos, como el japonés, en el que la ortografía francesa, tan alejada del francés oral, hace que los estudiantes transcriban lo que escuchan en katakana o rômaji, al lado de la escritura latina (Detey, 2007).
} 
Por su parte, el francés oral complementa el vocabulario y la gramática con un sistema fonológico rico en variantes combinatorias, y sobre todo con una prosodia fecunda, que posee valores semánticos precisos, y una sintaxis oral que delimita las categorías gramaticales y sintácticas de la frase: estos numerosos elementos tienen un valor semiológico y se añaden en cada acto comunicativo volviendo polisémicas las palabras orales. Estos componentes tampoco corresponden stricto sensu a los del francés escrito, aunque haya algunas equivalencias que pueden ser aprendidas independientemente.

Respecto a esta doble naturaleza de la escritura, nos parece primordial distinguir entre «escribir un texto en francés-escrito» (libros, novelas, cuentos, cartas, reportes, ensayos, "compte-rendus", disertaciones, etc.) y "poner por escrito palabras de francésoral». Esto se puede ver claramente en varias innovaciones tecnológicas de los últimos años (email, Messenger, chats, foros, Facebook, WhatsApp, etc.) que han plasmado en la cotidianidad y potencializado esta diferencia entre escribir (una lengua) y hablar por escrito (con letras). Espontáneamente, cuando se está aprendiendo a escribir su lengua materna, se empieza escribiendo como si se hablara, es decir que se utilizan las letras como código sustitutivo de los fonemas, y sólo un prolongado aprendizaje escolar les permite a los niños aprender realmente a escribir, es decir aprender la escritura como sistema semiológico autónomo.

Ahora bien, cuando se aprende una lengua extranjera, es fácil confundir estos dos tipos de escrituras, pues ambas tienen una función en el aprendizaje, pero, como ya se dijo, en diferentes momentos:

- Los sistemas escritos sólo se enseñan al final del aprendizaje, cuando la base idiomática ya está afianzada.

En cambio,

- el código sustitutivo se utiliza desde el principio del aprendizaje.

Retomemos entonces lo ya dicho según esta distinción semiológica: la escritura, es decir, las letras tienen ventajas innegables en el aprendizaje de una segunda lengua para los estudiantes que ya están alfabetizados en su lengua materna: las letras les dan cierta concreción a los objetos representados y, por lo tanto, permiten una mejor comprensión de las unidades lingüísticas (fonemas, monemas, sintagmas). El estudiante puede recordar mejor esas unidades y revisarlas si lo necesita. El medio visual le otorga igualmente autonomía al estudiante, quien incluso puede llegar a autocorregirse con la ayuda de un modelo escrito. Además, siendo un código que se puede utilizar al mismo tiempo que el código oral (uno puede oír algo y a la vez ver algo escrito), las letras aumentan la comprensión. Finalmente, el uso de las letras le permite al docente inventar gran variedad de ejercicios para un número considerable de estudiantes simultáneamente 
en el aula de clase, y reduce el tiempo que necesita para corregir a cada estudiante, actividad ardua y demorada cuando se trata de una corrección oral.

Estas ventajas de las letras como símbolos visuales parecen tener una incidencia en la supremacía que posee la escritura en el aprendizaje de las lenguas orales, pero es necesario entender que estas ventajas no son características exclusivas de la escritura latina del francés: todos los códigos sustitutivos visuales poseen estas ventajas.

Recordemos ahora la mezcla de lenguas que mencionamos al principio y que es un efecto negativo de la utilización de la escritura latina en el aprendizaje del francés de los estudiantes hispanohablantes, por la similitud de origen del español y del francés. En la didáctica del francés para hispanohablantes, las letras en alfabeto latino presentan entonces una gran desventaja frente a otros códigos sustitutivos visuales.

Ahora bien, buscando enseñar el francés oral antes que el francés escrito, descubrimos que casi no existe material didáctico oral que no esté combinado con la escritura latina. Sólo desde hace poco más de un siglo se empezó a grabar lo hablado, y este registro ha permitido utilizar en la enseñanza de lenguas el material sonoro, pero este siempre ha venido acompañado con un material escrito que lo complementa. Incluso cuando se trata de aprender los fonemas del francés, las palabras grabadas también aparecen escritas en los documentos audiovisuales. El problema que encontramos se puede entonces formular así:

¿Cómo eliminar esta desventaja de la escritura latina y evitar la mezcla de las letras francés-español en el aprendizaje temprano del francés?

Un código ad hoc para la didáctica del aprendizaje temprano del francés oral en hispanohablantes

El predominio de la escritura entre las poblaciones alfabetizadas es casi absoluto; fue sólo en el siglo XIX cuando por fin se le reconoció su lugar a la lengua oral con el nacimiento de la lingüística -el cual conllevó un enfrentamiento importante con los filólogos-. Muchos lingüistas intentaron entonces crear una notación específica de los sonidos de las lenguas (Malmberg, 1991; Passy, 1887) y este esfuerzo común, impulsado por el desarrollo de la fonética instrumental, dio como resultado la creación de numerosos alfabetos fonéticos y condujo a la creación del Alfabeto Fonético Internacional -AFI-, desarrollado a lo largo del siglo XX y que ya predomina en nuestro siglo digital, en el que se utiliza gran cantidad de códigos sustitutivos que se adaptan al funcionamiento informático (Asociación Fonética Internacional, 1999). 
Cuando en los años cuarenta se dio a conocer ampliamente la importancia de la estructura de los fonemas (Buyssens, 1980; Troubetzkoy, 1949)2, el AFI se empezó a utilizar de diferentes maneras en el aprendizaje de las lenguas (Chevalier, 1997; Suso, 2002). Luego, en reacción al estructuralismo rígido que le dio la exclusividad a esta estructura cerrada de los fonemas (Hjelmslev, 1971) en detrimento de la estructura abierta de los semas (Buyssens, 1943; Prieto, 1975b) y del contenido semántico y comunicativo (CaptArtaud, 1994), se fue abandonando el uso del AFI en la enseñanza de lenguas y, actualmente, es raro su uso en didáctica en nuestro medio ${ }^{3}$.

Tal vez como producto de esta historia del AFI, hemos podido observar que algunos de los que preconizan que se le otorgue un espacio más amplio a la enseñanza del francés oral apartan al mismo tiempo el AFI como instrumento didáctico (Baqué y Estrada, 2010), como si hubiera que eliminar todo lo visual para poder aprender lo auditivo. Para nosotros, es indispensable ofrecerles a los estudiantes las herramientas adecuadas que les permitan dominar el francés oral antes de iniciar el aprendizaje del francés escrito. Los alfabetos fonéticos, y en particular el AFI, permiten ejercer la memoria visual para retener los sonidos de las lenguas, pero, además, al ser el AFI diferente de la escritura latina, este no da lugar a mezclar las lenguas, y por tanto tiene en nuestra didáctica las ventajas de las letras latinas sin las desventajas de estas.

Es cierto que el AFI es actualmente un poco complejo, porque se aumentó con muchos detalles fonéticos que no siempre resultan pertinentes para un propósito pedagógico. Sin embargo, reducido a lo esencial, es un código sustitutivo de los fonemas de las lenguas que puede ser útil para dictar, desde el primer momento, una enseñanza exclusivamente oral, no sólo con material sonoro, sino también con un material mixto, sonoro y visual.

Desde luego, este uso del alfabeto fonético exige un esfuerzo por parte del estudiante, pero tiene sus ventajas, por ejemplo, dominar bien este alfabeto desde el principio es provechoso para el aprendizaje ulterior pues los diccionarios actuales contienen en su mayoría la transcripción fonética de las palabras y conociendo este alfabeto el estudiante puede aprender por sí mismo la pronunciación de las palabras desconocidas. Más tarde, todos los aprendizajes se vuelven más fáciles con esta base. Asimismo, poder pronunciar y escuchar correctamente le da al estudiante gran seguridad

\footnotetext{
2 Señalamos que el trabajo de los fonólogos fue el aporte fundamental para la revolución informática, pues fueron ellos quienes mostraron que la estructura de los fonemas era negativa, relativa y oposicional, y que las lenguas podían entonces verterse en un código binario. Es ahora que podemos ver en lo cotidiano las nociones de "sistema" y de "oposición" que la lingüística desarrolló en el siglo XIX (Saussure, 1879).

${ }^{3}$ En China, sin embargo, todavía se utiliza de manera muy normativa, siguiendo la tradición rusa, y se encuentra otro tipo de dificultades por el contraste entre el sistema alfabético del AFI con el sistema silábico del chino, pues un alfabeto fonético como sistema sustitutivo no es muy ventajoso para los estudiantes chinos (Li, Yin y Pu, 2018).
} 
respecto a sus conocimientos y su saber-hacer, lo que le permite seguir aprendiendo sintiendo placer, lo que potencializa el aprendizaje posterior.

Por otra parte, es más fácil exigir este trabajo al principio del aprendizaje, cuando los estudiantes están entusiasmados y dispuestos a esforzarse para enfrentarse con lo desconocido. Al contrario, unas bases orales débiles pueden retrasar el progreso e incluso causar estragos pedagógicos importantes, desanimando a los estudiantes, aún a los más aplicados. También hemos observado que el factor lúdico de los ejercicios que utilizan este AFI simplificado es muy positivo para el aprendizaje, pues no se trata de aprender "la fonética" y todo el aparato especializado de los fonetistas, se trata, únicamente, de un pequeño juego en otro alfabeto, como cuando se aprende el alfabeto griego o cirílico y se escribe el español con estos alfabetos. No es más que una diversión que no exige mayor esfuerzo intelectual y que puede ser aprendido con placer.

Ahora bien, como ya se dijo, no hemos encontrado material didáctico oral que no involucre las letras latinas; por eso tratamos de construirlo con la ayuda del AFI.

\section{Herramientas pedagógicas de análisis semiológico}

El uso del AFI fue presentándose en nuestra práctica pedagógica porque, poco a poco, con la ayuda de las nuevas tecnologías informáticas, hemos ido introduciendo unas herramientas particulares, que hemos llamado prácticas de transferencia y prácticas de contraste, y que pasamos a describir a continuación.

\section{Las prácticas de transferencia}

La noción de práctica en semiología fue definida por Prieto (1975a), primero a partir de la noción de praxis utilizada en los trabajos sociales influenciados por el marxismo del siglo XX. Sin embargo, Prieto limita esta noción al generalizarla en relación a la teoría de Buyssens (1943) sobre la comunicación y al trabajo de Troubetzkoy (1949) en fonología. Llega así a definir la comunicación como la práctica social que le otorga la pertinencia fonológica a los fonemas construidos por los hablantes. Con esta noción de práctica, Prieto continúa además la elaboración de Saussure sobre la pertinencia del punto de vista que crea las unidades semiológicas, en particular el signo lingüístico, dándole una solución epistemológica decisiva a lo planteado por Saussure y Trubetzkoy, como lo explicó Mejía en su artículo Unde exoriar? (1997). Más adelante, al trasponer esta noción de práctica lingüística al campo más extenso de la semiología, Prieto (1991) abre la noción distinguiendo las prácticas materiales que tienen como fin un objeto material, de las prácticas simbólicas que tienen como fin un objeto simbólico. Fundamentadas en estas nociones, elaboradas por Prieto a lo largo de su obra, las prácticas de transferencia fueron definidas en 2010 de esta manera: 
Podemos definir a nivel general las prácticas de transferencia como aquellas prácticas en las cuales un objeto simbólico que es un fin que un sujeto alcanzó con un medio dado, otro sujeto intenta alcanzarlo con otro medio diferente. La lectura en voz alta de un texto, la escritura de un discurso oral, la transcripción de un manuscrito al código impreso, la traducción, son todas prácticas de transferencia en las que cambia el "medio" y el sujeto o, mejor dicho, cambia la identidad del sujeto. (Mejía y Marmolejo, 2010, p. 197).

En ese artículo, la palabra transferencia se toma en su sentido más general de traspasar algo de un lugar a otro, y no corresponde a un campo particular, pues se puede aplicar tanto a la transferencia en psicoanálisis como a la transferencia en informática. En las prácticas de transferencia se busca producir un resultado concreto, con la ayuda de un modelo que otro sujeto ha realizado espontáneamente, pero que difiere de este primer producto en una o más variables. Actividades como la transcripción o la traducción son prácticas de transferencia que hemos utilizado en clase, y que pueden tener muchas variantes: se puede transcribir en escritura un audio del francés (transcripción de un código oral al escrito), pero también transcribir un manuscrito a un texto impreso (transcripción entre dos códigos escritos, de cursiva a imprenta). La traducción también tiene varios tipos: se traduce de una lengua a otra, tanto entre lenguas orales como entre lenguas escritas, pero también se puede traducir un texto escrito a un discurso oral y de un discurso oral al texto escrito. La lectura es otra práctica de transferencia, también las copias de planos de arquitectura y de obras de arte, así como los ejercicios de imitación. Según nuestra experiencia, las prácticas de transferencia tienen un gran potencial pedagógico por varios motivos:

- Proponen un modelo al estudiante que le sirve de guía: este modelo es un producto acabado que el estudiante debe analizar, pues si quiere transferirlo debe entender en cierta medida cuál fue el proceso de quien lo hizo primero, y así poder hacer "lo mismo", aunque adaptándolo a su propósito. El estudiante debe analizar entonces el modelo para entender el proceder de la persona que lo realizó, discriminando los rasgos del producto pertinentes para él; este análisis le proporciona herramientas útiles para llevar a cabo su propio objetivo.

- Las prácticas de transferencia le permiten al estudiante ejercer su autonomía pues la única ayuda que recibe para realizar la transferencia es el modelo, lo que resulta en mayor confianza en sí mismo, cuando la transferencia es exitosa.

- Todo el proceso de transferencia implica adquirir bien un buen número de conocimientos de toda índole, que el estudiante va absorbiendo sin darse cuenta y que se memorizan de manera muy estable pues es el mismo estudiante, con su propia experiencia, quien los descubre. 
- Gracias a estas prácticas los estudiantes entienden la diferencia entre un producto y el proceso que se realizó para llegar a ese producto, lo que les permite ver y comprender su propio proceso de aprendizaje, y tomar perspectiva frente a sus propios productos lingüísticos.

- Las prácticas de transferencia más elaboradas, como la interpretación y la improvisación, le permiten al estudiante desarrollar además su capacidad creativa.

La práctica de transferencia que nos parece la más adecuada para el principio del aprendizaje del francés oral es la transcripción, en especial el paso de un discurso oral a un texto escrito.

A este respecto, hay que distinguir claramente entre la transcripción como práctica de transferencia y los dictados tradicionales, pues estos no se basan en el francés oral. En los dictados, se trata de pasar un texto escrito en escritura latina, que se lee, al código visual de la misma escritura, o sea que la "pronunciación" del texto no es sino un intermediario entre un texto escrito y otro texto escrito en el mismo código. En los dictados no se trabaja el francés oral sino el francés escrito (se dicta hasta la puntuación), pues no se trata de hablar sino de "pronunciar el francés escrito": quien dicta, no está hablando, ni necesariamente ha sido quien ha escrito el texto dictado. En cambio, la transcripción como práctica de transferencia exige el análisis del acto de quien realizó el producto que se quiere transferir. En nuestras transcripciones, se proponen audios y videos de discursos reales, que han sido producidos en diversos actos de comunicación determinados. Se trata entonces de transferir a un código sustitutivo visual el discurso oral que se registró en un audio o en un video.

\section{Las prácticas de contraste}

La palabra contraste es clave en la semiología saussureana y está vinculada al método comparativo, el cual fundó la lingüística como disciplina en el siglo XIX (Meillet, 1925). Según Saussure (citado por Mejía, 1997), el principio metodológico es la crítica comparativa y contrastar los puntos de vista es lo que permite realizar esta crítica a cabalidad: "Il n'y a d'abord que la critique comparative des points de vue" (p. 98). Hemos escogido esta palabra de contraste para distinguir las prácticas de transferencia de otras prácticas, usuales en la comunicación y que se les asemejan porque también involucran productos que se comparan. Sin embargo, a diferencia de las prácticas de transferencia, de esta actividad no resulta un nuevo producto, sino una re-elaboración de uno de los productos comparados. Evaluar, corregir, verificar, supervisar son algunas de las prácticas de contraste más usadas. La corrección y sobre todo la autocorrección son el núcleo del trabajo en traducción, pero también en las actividades creativas como el diseño (Mesa y Mejía, 2011), ya que estas prácticas de contraste permiten avanzar y comprender mejor el producto que se está re-elaborando. 
La base de las prácticas de contraste es que siempre se deduce algo gracias al contraste, lo deducido luego puede concretizarse en la modificación de uno de los productos comparados; de esta manera el resultado puede ser una idea, es decir ese algo resultante del contraste, pero también un producto material donde se ha concretizado la idea. En este sentido, deducir a partir de una comparación se podría considerar como una práctica de contraste, nos referimos en especial a lo que se ha llamado en matemáticas la regla de tres, y que, con el nombre de formación analógica, fue presentada por Saussure (2019) como el principio general de las creaciones de lengua. En ese sentido, la regla de tres aplicada al funcionamiento idiomático debería ser la actividad principal en el aprendizaje de una segunda lengua ${ }^{4}$.

Las prácticas de contraste permiten desarrollar la capacidad de análisis y deducción, así como el espíritu crítico de los estudiantes frente a sus logros y errores. También les proporcionan una medida del tiempo de los procesos humanos $\mathrm{y}$, al darles seguridad, les permiten la libertad necesaria para la creación de todo tipo de objetos, físicos y mentales.

Aunque como procesos creativos las prácticas de contraste puedan dar lugar a actividades autónomas, generalmente no constituyen un todo entero como las prácticas de transferencia; más bien forman parte de otra actividad y a menudo se insertan en las prácticas de transferencia como una etapa adicional que permite perfeccionar la transferencia.

Las prácticas de contraste que más hemos utilizado en nuestra docencia del francés son la autocorrección y la deducción básica del sistema lingüístico.

\section{Metodología}

Durante varios años hemos utilizado las prácticas de transferencia y de contraste en el aula de clase de manera intuitiva, guiándonos por los buenos resultados observados en los estudiantes, mas, al querer involucrar de manera sistemática el AFI en las transcripciones, sentimos la necesidad de darle mayor coherencia a nuestro proceder $\mathrm{y}$, para evaluar metódicamente la eficacia de este alfabeto en el aprendizaje temprano del francés oral, planteamos la realización de una prueba que uniera nuestras herramientas en una propuesta metodológica armonizada.

\section{Propósito}

El propósito inicial fue observar detenidamente, desde un punto de vista semiológico, las ventajas y desventajas del uso de este código sustitutivo de los sonidos del francés en el aprendizaje temprano de esta lengua. La hipótesis que teníamos era la posibilidad de neutralizar con el AFI la influencia negativa de las letras latinas. Sin embargo, la prueba también incluía la evaluación metódica de las prácticas de transferencia y las de contraste,

${ }^{4}$ A este respecto, podríamos caracterizar nuestra perspectiva metodológica dentro de un enfoque analítico. 
pues, aunque ya conocíamos la eficacia pedagógica de estas herramientas, quisimos medir su alcance al combinarlas, así como la coherencia del conjunto.

\section{Grupo}

El grupo de esta prueba estuvo constituido por 10 estudiantes, todos principiantes, la mayoría no había tenido ningún contacto anterior con el francés. Los estudiantes estaban inscritos en el programa de Traducción Inglés-francés-español de la Escuela de Idiomas de la Universidad de Antioquia y especialmente en el curso de Francés I y aprestamiento a la traducción (semestre 2017-2). Este grupo siguió un curso de 8 horas a la semana durante 15 semanas.

\section{Material}

El material que se utilizó fue el siguiente:

- Tobón, A. A., Mesa, R. L., Rodas, L. P., y Zuiderduijn, C. (2008). Prononciation française pour hispanophones. Universidad de Antioquia.

- Audios complementarios de este libro: 006 - Unité 2 - Exercice 1.mp3.

- Videos de pronunciación de los sonidos del francés y sistema de transcripción de caracteres latinos a AFI del sitio web: easypronunciation, de acceso libre en ese momento. https://easypronunciation.com/es/french-phonetic-transcriptionconverter.

- The University of Iowa: Sounds of Speech: http://soundsofspeech.uiowa.edu/index.html\#english.

- Teclado para transcribir en API: http://www.typeit.org.

Siete extractos de entrevistas:

- Apostrophes, emisión de televisión presentada por Bernard Pivot, el 4 de mayo de 1984, en la cadena Antenne 2. Invitado: Claude Lévi-Strauss. Minutos: 36:10 - 37:55 y 38:05 - 38:59.

- "Mais qu'est-ce qu'ils pensent?" Emisión de Jean-Christophe Camus, en 1997. Invitado: Paul Ricoeur. Minutos 0:22 - 2:15.

- Entrevista realizada por Daniel Costelle el 9 de noviembre de 1965 como parte del programa de televisión Lire. Invitado: Georges Perec. Minutos 0:37 - 2:10.

- Entrevista realizada a Albert Camus por Jean Mogin el 13 de septiembre de 1955. Minutos 1:28 - 3:41.

- Fictions: Lectures d'enfance, programa de radio presentado por Marguerite Gateau, redifusión del 3 de julio de 2017. Emisión de France Culture. Minutos 1:13 - 1:51 y 24:24 - 25:26: La guerre des boutons de Louis Pergaud. 
- Fictions : Lectures d'enfance, redifusión del 7 de julio de 2017. Emisión de France Culture. Invitado: Anne Wiazemsky sobre La Belle et la Bête. Minutos 7:49 - 8:51.

- Les masterclasses, programa de radio presentado por Arnaud Laporte, el 4 de julio de 2017. Emisión de France Culture. Invitado: Christian Boltanski: Être artiste, c'est aussi une manière de guérir. Minutos: 16:04 - 16:46.

Seis extractos de videos publicitarios:

- OUI.sncf canal de Youtube de La Société nationale des chemins de fer français muestra una guía turística de Ginebra presentada por Alex V, publicada el 28 de febrero de 2013. Minutos 0:25 - 2 :16: Visite de Genève.

- OUI.sncf, guía turística del centro de Marsella presentada por Mathieu, publicado el 4 de octubre de 2011. Minutos 0:00 - 2:04: Vidéo Marseille : visite guidée de la ville.

- OUI.sncf, guia turística de Marsella presentada por Mathieu, publicada el 4 de octubre de 2011. Minutos 0:00 - 1:18: Vidéo Les calanques de Marseille à Cassis.

- Gouvernement de Monaco. Video publicado el 30 de septiembre de 2014. Minutos 0:00 - 1:16 y 2:25 - 3:43: Destination Monaco.

- Élection Montréal 2017 - Comprendre ma ville. Video publicado el 27 de noviembre de 2017. Minutos 0:00 - 2:48.

- Montréal, fière de ses racines autochtones MTLVille. Video publicado el 13 septiembre de 2017. Minutos 0:00 - 2:01.

Extractos de dos videos documentales:

- Le Pays des lémuriens - Madagascar - Fenêtres sur le Monde - Documentaire. Croisières et Découvertes - Documentaires canal de Youtube que presenta documentales de diferentes lugares del mundo. Video publicado el 08 de noviembre de 2013. Minutos 0:00 - 1:44.

- Bruges, une ville d'art - Belgique Croisières et Découvertes - Documentaires. Video publicado el 1 de marzo de 2014. Minutos 0:00 - 1:34.

Extractos de un discurso político y de dos monólogos:

- Charles De Gaulle, paroles publiques - Appel du 22 juin 1940 - Ina.fr. Minutos 0:00 $-2: 03$.

- Brèves histoires de la culture, programa de radio presentado por Jérôme Clément, el 18 de julio de 2017. Emisión de France Culture. 1792: La Marseillaise. Entero.

- Jean Marais raconte son métier d'acteur. Archivo del Ina. Minutos 2:33 - 2:57. 
Extractos de una película y dos piezas de teatro:

- Persepolis (2007). Adaptación cinematográfica del libro de Marjane Satrapi. Minutos 1:30 - 2:55.

- Camoletti M. (1993). Sexe et jalousie - Pièce de théâtre de boulevard. Minutos 2:00 2:34.

- Italie-Brésil 3 à 2, adaptación de la obra de Davide Enia. Emisión de France culture del 9 de julio de 2011. Fictions / samedi noir, programa de radio presentado por Alexandre Plank, el 9 de julio de 2011. Minutos 2:36 - 3:08 y 44:36 - 48:07.

Extracto de la lectura de dos libros:

- Carnet nomade, programa de radio en el que Colette Fellous realiza la lectura de Les mystères de Paris en el marco del programa Traversée de Paris. 3 primeros minutos.

- Lectura de L'Étranger de Albert Camus, leído por el autor. Minutos: 0:00- 1:00.

Tres canciones:

- Joe Dassin.(1995). Alors qu'est-ce que c'est? Joe Dassin [CD]. Collumbia.

- Mickey 3D. (2003). Respire. [CD]. Virgin Records.

- Aznavour, C. (1965). La Bohème. [CD]. Barclay Records.

Textos en códigos escritos:

- Transcripciones en AFI de 18 audios propuestos.

- Transcripciones en escritura latina de todos los audios, canciones y videos propuestos.

- Traducción de 2 transcripciones en escritura latina.

- Un cuadro sinóptico de gramática en AFI (artículos y pronombres).

- 2 ejercicios sobre artículos en AFI.

\section{Las herramientas pedagógicas}

El material sonoro se utilizó con variantes de las prácticas de transferencia y las de contraste. La transcripción tenía varias secuencias: generalmente, como primer paso pedimos transcribir el discurso oral al código sustitutivo del AFI, pero en un estadio ulterior, también se transcribió entre los dos códigos visuales, del AFI a la escritura latina, con ayuda del audio y al final del curso ya se transcribía directamente en escritura latina. La transcripción permite también crear ejercicios de rellenar espacios en blanco, los cuales se utilizaron según los contenidos que se quería introducir (terminaciones verbales, sufijos de sustantivos y adjetivos, sintagmas nominales o verbales, pronombres complementos, etc.). 
Prácticas de transferencia utilizadas, en orden cronológico:

- Se pidió a los estudiantes que transcribieran un audio al AFI, sin tener ningún conocimiento del sentido de lo que se decía, como en el siguiente extracto (Figura 1):

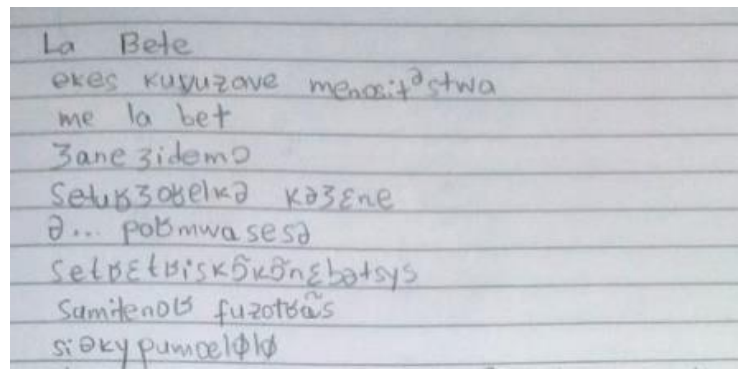

Figura 1. Ejemplo de transcripción en AFI sin conocer el sentido del texto

- Se pidió a los estudiantes que transcribieran el audio de un video al AFI, sin tener ningún conocimiento del sentido de lo que se decía, pero con la ayuda de las imágenes del video.

- Se pidió a los estudiantes que transcribieran partes de un audio al AFI: tenían que rellenar blancos, teniendo buena parte del audio escrito en AFI, como en el siguiente extracto en el que la transcripción del estudiante se encuentra en amarillo

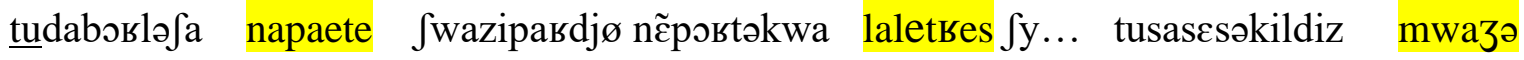

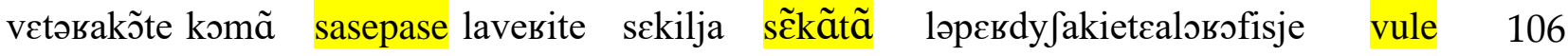

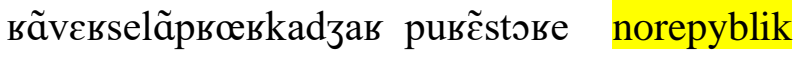

- Se pidió a los estudiantes que se grabaran leyendo un texto en AFI, después de oír el audio correspondiente.

- Se pidió a los estudiantes que transcribieran un texto AFI a la escritura francesa, sin ayuda de diccionarios, y con el audio correspondiente, como en la Tabla 1.

Tabla 1. Ejemplo de una transcripción

\begin{tabular}{|c|c|}
\hline 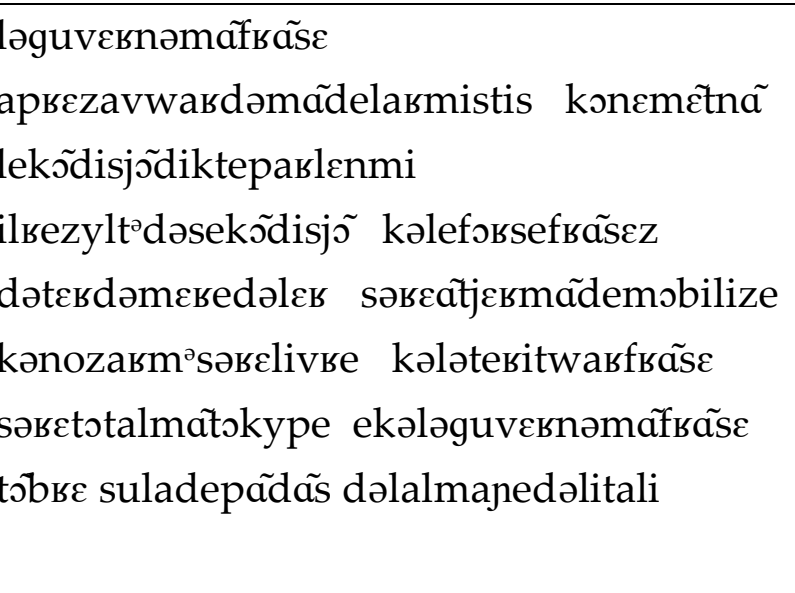 & $\begin{array}{l}\text { Le gouvernement français aprais avoir } \\
\text { demandé l'armistis. Conait maintenant } \\
\text { les conditions dicté par l'enmi. Il resulte } \\
\text { de ses conditions que les forces } \\
\text { françaises de terre, de mair et de l'air } \\
\text { seraient entierment demobilise. Que nos } \\
\text { arme serait livre que le territoire français } \\
\text { serait totalment ocupé et que le } \\
\text { gouvernement français tomberait su la } \\
\text { dependance de l'almagne et de l'Italie. }\end{array}$ \\
\hline
\end{tabular}


- Se pidió a los estudiantes que transcribieran un texto AFI a la escritura francesa, con ayuda de diccionarios en línea, y con el audio correspondiente.

- Se pidió a los estudiantes que transcribieran partes de un audio a la escritura francesa directamente, teniendo la mayoría del texto en francés escrito.

- Se pidió a los estudiantes que transcribieran un audio entero a la escritura francesa directamente, como en el siguiente extracto

Mateu: Bonjour, la citée focéenne nous accueille aujourd'hui sous un soleil éclatant. Je me trouve actuellement sur le vieux port qui a servi de décore au film de Marsel Pagnol. On va partir tout de suite pour le Panier, le quartier historique de Marseille qui est juste derrière moi. Sylvie sera notre guide aujourd'hui dans le Panier, alors racontez-nous un peu ce quartier de Marseille

- Se pidió a los estudiantes que tradujeran al español un texto que ya habian transcrito en AFI y en escritura francesa (Tabla 2).

Tabla 2. Ejemplo de la traducción de una transcripción

\section{- Vous avez sonné ?}

- Ben oui, puisque vous êtes là

- Ah oui, en effet

- Bon... alors, si je vous dis « samedi », qu'est-ce que ça vous dit?

- Ça me dit rien du tout

- Comment ça, rien du tout?

- $\quad$ Et bien oui, si vous me dites «ça me dit » sans dire ce qui vous dit, je ne peux pas savoir ce que ça vous dit

- Ça me dit pourquoi ?

- Je ne sais pas, moi, pour manger, ou pour autre chose

- Ben non, je dis « samedi »,

- Oui, ça vous l'avez dit

- Mais quand je vous dis « samedi », je vous parle du jour !

- Ah ! du jour, alors oui, çamedit comme ça, samedi, enfin ça me dit que c'est samedi, mais ça, je le savais déjà

- Bon, d'accord.

\section{- Has tocado?}

- Bueno sí, es que estás allí

- Ah sí, en efecto

- Bueno, entonces, si te digo "me suena", ¿qué te dice eso?

- Eso no me dice nada de nada

- ¿Cómo así, nada de nada?

-Bueno, sí, si me dices “me suena” sin decir qué es lo que te suena, no puedo saber a qué es que te suena.

-¿"Eso me suena" por qué?

-No sé, yo, por comer o por otra cosa -bueno, no, yo dije "me suena"

-Si, tu lo has dicho

- Pero, cuando te digo "me suena" voy a hablar de el día

- $\quad$ Ah, del día, entonces, eso "me suena" como "me suena", en fin, eso me dice qué es "me suena", pero eso, ya lo tuve.

- De acuerdo 
Prácticas de contraste utilizadas:

Autocorrección: Los estudiantes corrigieron ellos mismos todas las transcripciones, con la ayuda del modelo que se entregó cada vez al finalizar la transferencia. Asimismo, se les pidió realizar un análisis detallado de los errores que habían cometido: el estudiante señalaba el error y trataba de explicitar por qué era un error y cómo lo había cometido. Estas son las variantes de la autocorrección que se utilizaron:

- Se pidió a los estudiantes que se autocorrigieran con ayuda de la transcripción que se les entregó. Ellos mismos debían encontrar los errores que habían cometido comparando su versión con la entregada por el docente, como en este extracto (Tabla 3):

Tabla 3. Ejemplo de autocorrección

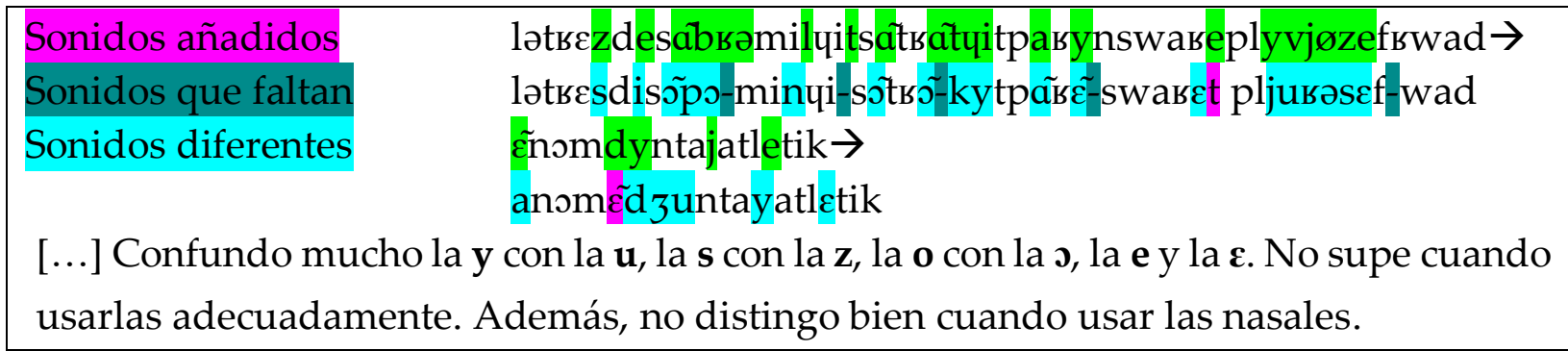

- Se pidió a los estudiantes que explicaran las diferencias entre su transcripción y la transcripción modelo, según las diferencias que marcaba el comparador automático del programa Word.

En ambos ejercicios debían tratar de entender tanto el error en el producto como el error de proceso.

Heterocorrección: Antes de las autocorrecciones correspondientes se realizaron correcciones por pares en dos ocasiones. A veces la transcripción "modelo" presentaba errores, y cabe anotar que siempre esos errores fueron señalados por alguno de los estudiantes, que de esta manera también corrigieron lo presentado por el docente. Una vez se les propuso una transcripción con varios errores adrede que debían encontrar. Al final del proceso de autocorrección, los estudiantes recibían asimismo la revisión de su autocorrección con comentarios del docente y consejos para entender mejor los errores de producto y, sobre todo, los de proceso y cómo evitarlos.

Deducción. Se propusieron actividades como:

- Se pidió a los estudiantes que, a partir de las transcripciones en AFI y las transcripciones en escritura francesa que se les entregaron de los mismos audios, dedujeran las 
correspondencias entre los fonemas del AFI y las letras del francés, como se muestra en la Tabla 4:

Tabla 4. Ejemplo de deducción de correspondencias entre fonemas y formas escritas del francés

\begin{tabular}{|c|c|}
\hline Vocales & Escritura francesa \\
\hline$\partial$ & e, ue (Ej : que, le, de) \\
\hline$\varepsilon$ & e, ais, è, aît, ai, aient, ait, est (Ej : français, seraient, \\
connaît, qu'est-ce que c'est?)
\end{tabular}

- Se pidió a los estudiantes que, a partir de listas de verbos conjugados que se les entregaron, dedujeran las terminaciones de personas y de tiempo verbales.

Se propusieron también actividades de reconocimiento de categorías sintácticas:

- Se pidió a los estudiantes que, gracias al análisis de los monemas y de entonaciones de los grupos fónicos, señalaran con colores en los audios transcritos diferentes categorías sintácticas (pronombres, verbos, grupos nominales, preposiciones, adverbios, etc.), como en este extracto (Tabla 5):

Tabla 5. Ejemplo de reconocimiento sintáctico y análisis verbal de una transcripción

Qui êtes-vous? Georges Perec

Et bien, j'ai 30 ans, effectivement. Je suis documentaliste dans un laboratoire de recherche medicale, c'est-à-dire que je reçois des articles en anglais, en italien ; je fais des fiches et j'élabore tout un système de bibliographie qui sert à la recherche. C'est un travail que je fais depuis 4 ans, qui me prend la plupart de mes journées, mais je parviens à reserver quelques heures, en fin d'après-midi ou le soir pour écrire. Je suis marié, depuis 5 ans, mais je n'ai pas d'enfants.

\begin{tabular}{|c|c|c|c|}
\hline Verbe & Personne/Genre & Mode & Temps \\
\hline êtes & $2^{\text {ème }}$ Pluriel & Indicatif & Présent \\
\hline
\end{tabular}




\begin{tabular}{|c|c|c|c|}
\hline ai & $1^{\text {ème }}$ singulier & Indicatif & Présent \\
\hline suis & $1^{\text {ème }}$ singulier & Indicatif & Présent \\
\hline est & $3^{\text {ème }}$ singulier & Indicatif & Présent \\
\hline dire & & Infinitif & \\
\hline reçois & $1^{\text {ème }}$ singulier & Indicatif & Présent \\
\hline
\end{tabular}

- Para las canciones, que también se pidió transcribir, se realizaron además actividades de reconocimiento de prosodia y cortes sintagmáticos correspondientes a los grupos fónicos, en especial con la canción de Mickey 3D 5 .

Todos los productos de los estudiantes fueron revisados por el docente y, para aquellos en los que la autocorrección produjo numerosas preguntas, se envió retroalimentación personal por correo electrónico. Posteriormente, cuando ya todos los estudiantes habían realizado sus autocorrecciones en casa, la actividad se volvía a revisar en el aula de clase entre todos, y el docente daba entonces retroalimentación sobre los errores comunes y contestaba las preguntas que surgían en el grupo y que fueron de todo tipo (gramaticales, lexicográficas, traductoras, culturales, geográficas, lingüísticas, etc.). Durante estas retroalimentaciones, y con los ejemplos de las transcripciones mismas, se introducían y enfatizaban los contenidos gramaticales correspondientes al curso. En los ejercicios de deducción se afianzaron y matizaron las reglas deducidas (correspondencias entre fonemas y letras, categorías gramaticales y sistema verbal).

Además, estas herramientas se complementaron con ejercicios tradicionales sobre los contenidos del curso, en especial ejercicios sobre tiempos verbales simples, el passé composé y sus diversas concordancias, y los verbos semi-auxiliares, acompañados de las correspondientes heterocorrecciones, autocorrecciones y retroalimentación.

\section{Desarrollo del curso}

El curso siguió el siguiente orden durante cuatro meses:

El primer mes se trabajó esencialmente el reconocimiento de los fonemas y grupos fónicos del francés, gracias a la transcripción en AFI, primero de audios, luego de videos. Esto se complementó con un trabajo de articulación de los grupos fónicos con las grabaciones de las transcripciones en AFI. Y se empezó con el reconocimiento de las categorías sintácticas básicas en las transcripciones gracias a la prosodia (artículos,

${ }_{5}^{5}$ Este ejercicio se adaptó del propuesto por Philippe Mijon en su página web FLE Philippe Mijon: http://www.fle-philippemijon.com/materiel-didactique/accentuation-phonetique-en-francais-regles-etactivite/. 
sustantivos, verbos y pronombres), además de introducir los contenidos correspondientes (conjugación presente y subunidades morfológicas).

El segundo mes se trabajó la escritura francesa, con ejercicios de deducción de correspondencias entre el AFI y la escritura en caracteres latinos, y transcripción entre ambos códigos visuales. Se presentó la organización del diccionario monolingüe en línea del Centro Nacional de Recursos Textuales (CNRTL) y otros instrumentos de esta página. Se desarrolló la escucha de los sintagmas fónicos y su escritura en varias palabras, y se introdujeron nuevas categorías gramaticales (preposiciones, adjetivos y adverbios). Se continuaron las grabaciones de los textos en AFI. En cada clase se presentaban además los contenidos gramaticales y de vocabulario necesarios, en especial con la revisión de las autocorrecciones de ejercicios tradicionales.

El tercer mes se dedicó al sistema verbal que se presentó en conjunto, con actividades de deducción y reconocimiento de verbos en los audios y videos transcritos, y se afianzaron las actividades precedentes. Después de la deducción del sistema verbal, se realizaron varios ejercicios escritos tradicionales de los tiempos simples en especial de los irregulares, pero con su respectiva autocorrección y revisión. Se añadió al final la traducción de dos videos transcritos.

En el cuarto mes, más corto que los demás, se afianzó el sistema verbal y se desarrolló el producto del proyecto de aula de los estudiantes, estipulado en el programa

111 del curso, que comprendía como actividad final del semestre la exposición de un tema escogido por los estudiantes: a partir de los textos y la información que habían buscado durante el semestre, escribieron un discurso de entre 5 y 10 minutos que prepararon de antemano y que dijeron ayudándose con una presentación visual.

\section{OBSERVACIONES}

Lo observado en el aprendizaje de los estudiantes fue bastante positivo, sólo se presentó un punto parcialmente negativo que atañe a un factor psicológico.

\section{Grupo}

En el curso se inscribieron 21 estudiantes. Es común que después de las dos primeras semanas algunos estudiantes cancelen la asignatura por diversos motivos (en general incompatibilidad horaria con el trabajo $\mathrm{u}$ otros estudios, menos frecuentemente enfermedad o desplazamientos). Por esto, aunque la propuesta metodológica se presentó desde la primera clase, sólo firmaron el consentimiento informado los 16 estudiantes que quedaban en la tercera semana. De estos, cuatro estudiantes se retiraron antes del corte del $40 \%$ de evaluación, momento en el que ya no pueden retirarse voluntariamente si tienen una evaluación negativa y dos estudiantes menores de edad quedaron de entregar el consentimiento firmado por sus padres, pero nunca lo hicieron. Los trabajos de diez 
estudiantes durante todo el semestre constituyen pues el material que analizamos en esta prueba.

\section{Metodología implementada}

Con respecto a la metodología implementada en el curso, cabe anotar que esta se les explicó a los estudiantes al principio del semestre y luego se les entregó el consentimiento informado que todos firmaron aceptando que se pudieran utilizar y publicar sus trabajos escritos. Durante el semestre, se les dio retroalimentación frente a los objetivos de las actividades que se les presentaban, y también se les compartieron las observaciones parciales. Mensualmente se hizo una asesoría personalizada de evaluación con cada estudiante, tanto de su desempeño como de la metodología implementada, y en todo momento se les hizo partícipes, pidiéndoles expresarse sobre cada actividad propuesta.

Los efectos fueron bastante variables en el aprendizaje de los estudiantes: el progreso de cada uno y la intervención del docente fue muy diferencial, según cada estudiante. Con los más autónomos se llegó a explicar muchos temas más de lo contemplado en el programa de la asignatura, a nivel gramatical y cultural; con los demás se cumplió lo previsto en el programa, y con algunos se necesitó mucho trabajo de apoyo en asesorías personales y explicaciones básicas para que asimilaran el mínimo requerido. Esta variabilidad no es de extrañar, pero vale la pena recalcar que la metodología permitió que se siguiera a cada estudiante según su ritmo y sus progresos, sin ningún esfuerzo particular por parte del docente. Se pudo realizar un trabajo personalizado con cada estudiante, sin implementar ninguna estrategia diferente de la metodología general.

A pesar de lo variado de los progresos de los estudiantes, hubo un núcleo común a todos, que fue el punto positivo más importante de la prueba y que reforzó la hipótesis de la neutralización de la mezcla de lenguas, a saber, la buena escucha y pronunciación que todos desarrollaron, aunque, desde luego, en diferentes grados según cada estudiante.

\section{Material}

El único material que no tuvo éxito fue el cuadro sinóptico y el vocabulario en AFI. Al principio habíamos pensado realizar un pequeño manual de gramática simple escrito en AFI, pero rápidamente nos dimos cuenta de que no era rentable pues representaba demasiado trabajo para el docente en contraste con el poco uso que se le dio. Por esto, reservamos el uso del AFI estrictamente a las transcripciones de los audios del primer mes, enlazándolo siempre con las realizaciones orales. Con respecto a este uso, que sí tuvo éxito, señalamos que fueron los mismos estudiantes quienes propusieron la página web que permite escribir cómodamente el AFI.

Con los audios se obtuvieron excelentes resultados, aunque con gran variabilidad según los estudiantes. Unos cuantos terminaron entendiendo muy bien, y uno casi 
perfectamente, la mayoría aprendió a distinguir de manera satisfactoria los sintagmas fónicos del francés y sólo cuatro conservaron dificultades de escucha. Los audios se escogieron según las necesidades del programa del curso y del Proyecto de aula, el tema del audio no planteó ninguna variable sobresaliente, la edad de quienes hablaban en el audio tampoco. En cambio, observamos que la calidad del sonido, y algunas veces la claridad de la dicción de la persona (como la muy clara de Claude Levi-Strauss), más que la rapidez del discurso (los estudiantes podían disminuir la velocidad del audio para escucharlo mejor y cuantas veces quisieran) fueron los elementos más importantes para el éxito de la transcripción. Los videos tuvieron muchísima acogida pues las imágenes les ayudaban a tener una idea del sentido de lo que se decía; la obra de teatro en particular gustó mucho.

\section{Herramientas de análisis semiológico}

Con respecto a las prácticas de transferencia, y en particular a las transcripciones al AFI, los resultados fueron muy prometedores. Después de un mes los estudiantes asiduos empezaron a distinguir, entender y hasta a pronunciar bastante bien los fonemas y grupos fónicos. Esto es supremamente alentador cuando uno ha visto cómo después de dos, tres y hasta cuatro años de estudios los estudiantes que no han hecho ningún intercambio en un país francohablante siguen pronunciando mal el francés y poco entienden de los discursos espontáneos, aunque aparentemente entiendan los textos. Los problemas más importantes fueron, como era de esperar, los fonemas muy diferentes del español, las diferentes " $\mathrm{e}$ " francesas y las nasales, pero la escucha terminó siendo muy buena, según este nivel debutante.

Después del segundo mes, en el que se introdujeron las letras latinas, se abandonó gradualmente el AFI y se pasó a transcribir según las letras del francés, pero sin insistir en la ortografía correcta de la palabra, lo que lograron hacer con relativa facilidad, aunque según las inevitables variaciones personales. Desde el tercer mes, ya se pidió que buscaran en diccionarios y tuvieran cuidado con la ortografía de las palabras, pero siguió insistiéndose en la escritura sustitutiva.

Como punto negativo de las transcripciones de AFI a caracteres latinos, resaltamos que hubo al principio algunos "cortos circuitos" cuando los grafemas del AFI y de la escritura latina eran iguales aunque el sonido no lo fuera, como ocurre con las sibilantes $\mathrm{s}, \mathrm{z}$, lo que dificultó la transcripción. Cabe señalar, sin embargo, que estos errores se autocorrigieron fácil y espontáneamente.

En general, las actividades de transferencia resultaron lúdicas, como ya lo habíamos observado, aunque según el compromiso con su aprendizaje $\mathrm{y}$, más particularmente, el estado matinal del estudiante (la clase era a las 8am) también pudieron resultar tediosas para algunos, al implicar gran concentración. 
Con respecto a las prácticas de contraste, el punto positivo más importante fue el alcance de la importancia didáctica de la autocorrección.

La autocorrección fue extremadamente eficaz pues observamos que, poco a poco, fueron los errores que los mismos estudiantes señalaron, tanto en la escucha como en la escritura, los que fueron disminuyendo gradualmente, y en algunos estudiantes desaparecieron por completo al final del semestre. Estas son algunas de las conclusiones de tres estudiantes en la primera transcripción (primera columna) y al final del semestre (segunda columna):

Estudiante 1.

Algunas veces escucho sonidos que no se pronuncian, especialmente al comienzo de una palabra. Me confundo con los sonidos $\mathrm{Y} / \mathrm{U}-3 / \mathrm{j}$. Aún no tengo clara la diferencia entre los diferentes tipos de E. Y me confundo con la a y la e nasal.

Estudiante 2.

1. Problemas para distinguir las vocales

y sus versiones abiertas.

2. No escucho bien las v. Las confundo con 3u.

3. Confusión entre combinación vocal + $\mathrm{n}$ con vocal nasal.

4. A veces no escucho las $\mathrm{z}$.

5. Confundo la y con sonidos con articulación de u.

Estudiante 3.

1. He notado que todavía confundo muchísimo $\varepsilon$, e y ə, creo que se me dificulta mucho porque en el español sólo utilizamos una y no estoy acostumbrada.

2. a veces escuchaba zə sólo como una $\mathrm{s}$

3. Agregué muchas в donde no iban y no escuché muchas 1 , me parece que las pronuncian muy suave.
Me confundo con la $\varepsilon$ y e. Sigo confundiendo la $\tilde{\varepsilon} y$ la a.

\section{OBSERVACIONES}

En general estuvo todo bien, a excepción de una palabra por consideraciones ortográficas (escribí connart en lugar de connard) y la tilde en république.

Retomar sintaxis. Principal error : se

Creo que la mayoría de errores fueron ortográficos, pero es algo que voy a adquirir con la práctica $\mathrm{y}$ con ejercicios y los pocos errores de sonido y código sustitutivo considero que fueron por falta de atención. 
La actividad de deducción de las correspondencias entre fonemas y letras (en ese orden, de los fonemas a las letras) fue supremamente útil, los estudiantes pudieron diferenciar entre la escritura latina correspondiente a los fonemas, y la ortografía de la palabra, lo que parece haberlos "liberado" frente a la caprichosa ortografía del francés. Los estudiantes que realizaron esta actividad empezaron a tener una buena ortografía, que sorprendió al docente, pues la ortografía francesa es difícil de aprender, aún para los francohablantes. Además, se dieron cuenta de muchos detalles de las equivalencias entre sonido y escritura que surgieron con sus preguntas, como las siguientes de una estudiante:

Análisis

¿Cómo sé cuándo se debe usar la e tildada?

¿Cuándo se usan las letras dobles (ejemplo: ss,cc)?

¿Cuándo se transcribe el sonido/s/ como t?

¿Cuándo se transcribe el sonido/i/ como y?

¿Por qué en API estas palabras (Actuellement/Allemagne/Seulement) no tienen el sonido de la e?

Como punto negativo hay que señalar que, durante el primero y parte del segundo mes, al no abordar el sentido de lo que oían, se creó una inseguridad semántica que fue muy difícil de sobrellevar por algunos estudiantes, que no lograban postergar la realización de su deseo de entender el sentido. Esta inseguridad semántica llegó a convertirse en un relativo rechazo a las actividades, en particular para cuatro estudiantes. Sin embargo, todos los estudiantes reconocieron los avances realizados en la escucha y algunos de los estudiantes reacios manifestaron que oyendo hablar nativos del francés en la ciudad "entendían" la lengua, es decir que oían y reconocían los grupos fónicos, aunque no entendieran el sentido de lo que decían. Cuatro estudiantes manifestaron pues un descontento frente a lo que todavía no se les ofrecía, pero no hubo ninguna crítica, sólo satisfacción por parte de todos, sobre lo que sí se les propuso. Sin embargo, el ansia de entender el sentido parece haber dificultado las autocorrecciones, pues en dichos estudiantes fueron menos minuciosas y por ende menos provechosas.

Un resultado positivo sorprendente, que se observó claramente en las exposiciones al final del semestre, fue la buena calidad de la pronunciación de los estudiantes, desde luego, teniendo en cuenta las consabidas variaciones personales. Dentro de estas variaciones, señalamos que los estudiantes que ya habían tenido alguna experiencia de "inmersión" en otra lengua, u otra variedad lingüística del español, fueron quienes aceptaron y aprovecharon mejor lo propuesto. La buena calidad de la pronunciación nos sorprendió porque durante el semestre no se enfatizó particularmente en la producción oral, sólo hubo en todo el semestre unos cuatro ejercicios de producción con las 
grabaciones de los audios, sin embargo, al final del semestre, la expresión de los estudiantes resultó mejor que la que hemos observado en otros estudiantes de niveles más avanzados. Por consiguiente, el trabajo de escucha parece haber influenciado de manera extremadamente positiva la producción oral.

El objetivo de mejorar las competencias orales desde el principio del aprendizaje se alcanzó indiscutiblemente, e incluso los estudiantes reticentes a las actividades expresaron espontáneamente por escrito los logros con respecto a la escucha del francés. La dificultad para aceptar postergar la comprensión semántica fue el obstáculo psicológico importante que encontramos, aunque únicamente en menos de la mitad de los estudiantes, y, si bien en buena parte es inevitable como reacción al efecto de inmersión de la metodología, esperamos poder entenderlo mejor en un futuro cercano y así encontrar la manera de reducirlo.

\section{Análisis lingüístico de lo observado}

Después de este primer semestre, y a raíz de las múltiples explicaciones de todo tipo que tuvimos que dar a los estudiantes en las clases de revisión en grupo, observamos un hecho que no habíamos vislumbrado de manera precisa con anterioridad: la implementación realizada se opone claramente a una consigna respetada en la mayoría de los demás métodos, a saber, la necesidad de una progresión en el contenido suministrado: todos los audios y videos eran complejos a nivel de la lengua, lo que creó un efecto de inmersión en donde todos los componentes de la lengua aparecen juntos y conectados. Observamos entonces que lo que planteamos con el fin de evitar los ejercicios artificiales, al utilizar un material real, obligó naturalmente a presentar los contenidos lingüísticos sin ninguna progresión.

A este respecto, observamos que esta complejidad idiomática de los audios no fue realmente molesta para los estudiantes, aunque una consecuencia psicológica de esta complejidad sí lo fue: lo que más les preocupaba, sobre todo a algunos estudiantes, era no entender el sentido preciso de los audios. Esto se podría concebir como la clara experiencia de la arbitrariedad de la lengua, de la "otredad" del francés frente al español y la consecuente frustración inicial de todo principiante. Con las transcripciones de los audios reales se creó pues el efecto de inmersión que inevitablemente confronta con la impotencia idiomática a quien quiere hablar y lo lleva al mundo infantil del "no saber", lo cual, para todos, es una experiencia frustrante que algunas personas soportan mejor que otras. Es para evitar esta frustración inicial que el material artificial de los manuales de lengua presenta los contenidos gradualmente, y es común que los profesores de hispanohablantes, para darle seguridad a los estudiantes, utilicen lo que mal se llama la "transparencia" de las palabras francesas escritas.

Sin embargo, a pesar de este aspecto negativo de la inseguridad semántica, el efecto de inmersión de nuestra metodología permitió otro aspecto positivo, pues pudimos 
adentrarnos bastante en las peculiaridades de la lengua y la cultura francesas, lo que desarrolló en los estudiantes la apertura a la variación, a la diversidad de pronunciaciones (jóvenes y viejos, hombres y mujeres, campesinos y citadinos, etc.), a la variedad de acentos (canadiense, suizo, belga, francés del sur, etc.), de niveles de lengua, etc. Con aspectos positivos y negativos a la vez, este aspecto del efecto de inmersión dificultó la adherencia de algunos estudiantes, pero a quienes pudieron postergar la realización de su deseo de entender les permitió crear una base acústico-articulatoria real para el francés oral, y les dio la oportunidad de extender el campo de lo posible.

En vez de una progresión en el contenido, nuestra propuesta resultó pues fundamentarse en una progresión de competencias combinadas, de comprensión y de producción, según este orden:

Primero, los estudiantes aprendieron a reconocer y producir imágenes acústicoarticulatorias, o según un término lingüístico, a reconocer los "aposemas" del francés, que son unidades sin sentido, es decir formas sonoras, sílabas y enlaces fónicos sin sentido. Esta unidad lingüística fue mencionada varias veces por Saussure en sus manuscritos cuando intentaba definir las unidades sincrónicas y diacrónicas de la lengua, y la concibe como inherente al discurso (Saussure, 1974). Mejía $(1999,2000,2004)$ precisó la definición del aposema como un "promedio acústico-articulatorio" y la presentó como la unidad del hablar, distinguiéndolo del sema (unidad de lengua sincrónica) y del sôma (unidad de 117 lengua diacrónica), nociones que Saussure había explorado sin llegar a proponer definiciones claras:

Aposème pourrait se limiter à renvoyer à ce qu'un sème donné forme dans un acte de parole déterminé: «Les sujets parlants n'ont aucune conscience des aposèmes qu'ils prononcent, pas plus que de l'idée pure d'autre part. Ils n'ont conscience que du sème. C'est là ce qui assure la transformation parfaitement mécanique de l'aposème à travers les siècles» (3315.2). Circonscrire l'aposème à cette acception permet de bien comprendre sa nature toute «physique» en tant qu'enveloppe phonique du sème. (Mejía, 1999, p. 249).

Los aposemas son indispensables para aprender bien cualquier lengua, y en particular constituyen la base de la sintaxis. Ahora bien, no es común que se enseñen los aposemas de las lenguas pues se tiende a pensar que, si se aprenden los "semas", unidades con sentido, es decir las unidades de lengua, ya se conocen los aposemas.

En efecto, los aposemas corresponden en parte a los semas, sin embargo, una particularidad de los aposemas es que pueden corresponder a varias unidades de lengua y por eso son la base de lo que se ha llamado la "homonimia", por ejemplo, el aposema /VER/en francés corresponde a varios semas con diferentes significados que se pueden expresar en español como "verde", "gusano", "vidrio", "vaso" y también corresponde al 
sema de una preposición de movimiento. El francés oral es supremamente polisémico, es decir que varios semas corresponden a un mismo aposema, pero además un solo aposema puede englobar varios semas, citemos por ejemplo la palabra francesa "concupiscence", una "palabra canguro" que les gusta mucho a los niños en la edad del descubrimiento sexual, puesto que el aposema entero también corresponde a cuatro semas intrigantes: con, cul, pisse, sens. Para los hispanohablantes esta polisemia del francés oral es difícil de oír y entender, porque hacerlo implica que se hayan construido los aposemas del francés. En este primer semestre fue esencialmente la creación de los aposemas del francés lo que exigió el principal esfuerzo, y fue muy satisfactorio observar que los estudiantes más asiduos los crearon de manera natural.

En el segundo mes se introdujo la escritura: primero las correspondencias entre los fonemas y las letras, después se distinguió entre la ortografía de la palabra y la escritura de los fonemas uno tras otro. Luego, insistiendo en la gran diferencia entre el francés oral y el francés escrito, los estudiantes asimilaron que los sintagmas del francés oral corresponden a varias palabras del francés escrito, y se empezó a crear la correspondencia con los sintagmas nominales y verbales más usuales, introduciendo la importancia de las concordancias de número y género.

Es decir que, a nivel lingüístico, se utilizó la escritura en caracteres latinos para introducir los semas complejos, en particular los sintagmas, y para crear los vínculos entre sintagmas y aposemas, que vinculan estos últimos a una red de semas con sus evocaciones semánticas.

Al final del semestre, aunque en una medida muy reducida, se propuso la traducción, lo que permitió introducir el sentido en comparación con el español, dándole lugar a la variedad lingüística del francés, tanto geográfica como temporal, y se pudo empezar a crear las asociaciones entre semas propias del francés, diferenciándolas con mucho cuidado de las del español. Nos parece que esta pequeña red de asociaciones de unidades de lengua correspondientes a unidades del hablar fue la que permitió la buena producción oral del final del semestre. Pudimos observar que, aún los pocos estudiantes que no "dijeron" la exposición final, sino que la "leyeron", estaban muy seguros de la pronunciación y no pronunciaban las concordancias "inexistentes".

Observamos que la construcción de las unidades sintagmáticas del hablar precedió entonces la construcción de las unidades más simples. Este orden de las unidades, de la más extensa a la menos larga, se repitió con cada transcripción, pero en espiral, precisándose cada vez mejor el reconocimiento de las unidades que se repetían. Este fue un resultado muy importante pues no sólo corresponde al aprendizaje natural de los niños francohablantes, sino que encontramos asimismo una consecuencia de la influencia de la escritura que no habíamos observado: la escritura francesa le hace pensar al estudiante que el francés tiene marcas de número y género, y le hace creer que las unidades más importantes del francés son las palabras. Por esto, leyendo, los estudiantes pronuncian eses que no existen en francés oral, $\mathrm{u}$, oyendo, entienden todo en singular, 
cuando la frase dicha está en plural. En realidad, en francés ya no hay marcas de número y género en las palabras, sólo hay marcas en los sintagmas. Las marcas de género y número del sintagma nominal, por ejemplo, las portan los artículos, los demostrativos y, desde luego, la "liaison", rara vez los sustantivos o los adjetivos. En francés contemporáneo, los sintagmas son las unidades más importantes de la lengua.

Sin embargo, como todas las escrituras que no se modifican para adaptarse a la evolución de la lengua correspondiente, la escritura latina del francés sí conserva en todas las palabras concordancias desaparecidas en francés desde hace unos siglos. Por la ausencia de marcas de género y número en todas las palabras del sintagma, el francés oral es supremamente polisémico, y la transcripción exige gran atención en este punto, pues en la escritura francesa las marcas sí se encuentran en todas las palabras, y sirven, por cierto, para desambiguar dicha polisemia.

Por lo tanto, la transcripción es, además, la mejor manera de ver, concebir, entender y dominar esta gran diferencia entre el francés escrito y el francés oral, que en el caso de la traducción es esencial para la lectura del traductor. Con las transcripciones, se introdujo pues la polisemia del francés oral y la diferencia entre el francés oral y el escrito con respecto a las conjugaciones verbales y las marcas de género y número. Este trabajo sobre las concordancias fue bastante soterrado, y se hizo sin que hubiera sido un objetivo pensado desde el principio ya que en general este tema se aborda más tarde en el aprendizaje. Sólo después de realizada la prueba, se pudo comprender lo logrado, al constatar la facilidad de algunos estudiantes para escribir las concordancias escritas, que son en general un dolor de cabeza para los estudiantes más avanzados.

Por último, señalemos que queda por observar el grado de permanencia a largo plazo de los aprendizajes que constatamos.

\section{CONCLUSIÓN}

En este artículo presentamos primero el problema del uso de la escritura latina para el aprendizaje temprano del francés en hispanohablantes, y explicamos el interés que tiene utilizar el alfabeto fonético internacional en vez de la escritura latina, así como empezar el aprendizaje únicamente por el francés oral. Para realizar esto presentamos la propuesta semiológica de distinguir dos aspectos de la escritura, el código sustitutivo y el sistema semiológico, y mostramos cómo el primero es el que se utiliza en el aprendizaje temprano y el segundo sólo en una fase posterior. A continuación, expusimos unas herramientas pedagógicas de análisis semiológico, a saber, las prácticas de transferencia y las prácticas de contraste, incluidas junto con el uso del AFI en una propuesta metodológica, que se describió luego de manera detallada, tal y como se implementó en un curso de 120 horas, con estudiantes debutantes de francés. Para terminar, se explicitaron las observaciones, 
positivas y negativas, que esta implementación permitió realizar y se analizaron desde un punto de vista lingüístico con base en la lingüística saussureana.

Concluimos que, por los puntos favorables para el aprendizaje del francés en los hispanohablantes que se detallaron en los resultados, esta propuesta merece ser conocida, replicada por otros equipos y discutida para precisar mejor su alcance y encontrar la manera de disminuir el efecto negativo sicológico en algunos estudiantes.

Para terminar, señalemos que los estudiantes nos solicitaron seguir con el segundo nivel y los aspectos positivos de la implementación de esta metodología también hicieron surgir el deseo de continuarla otro semestre más, y así poder trabajar mejor la introducción del sistema de semas y observar lo que podría dar la metodología con dos semestres seguidos. Asimismo, quisimos implementar de manera más sistemática la autocorrección, que había dado tan buen resultado. Este segundo semestre se realizó y fue muy fructífero, pero también bastante complejo, pues fue positivo en aspectos sorprendentes que merecen una descripción pormenorizada, por lo que dedicaremos un próximo artículo para presentarlo.

\section{REFERENCIAS}

Alcalde, N. (2011). Principales Métodos de Enseñanza de Lenguas Extranjeras en Alemania Revista de Lingüística y Lenguas Aplicadas, (6), 9-24. doi: 10.4995/rlyla.2011.878.

Asociación Fonética Internacional. (1999). Handbook of the International Phonetic Association: A Guide to the Use of the International Phonetic Alphabet. Cambridge: Cambridge University Press.

Baqué L., y Estrada, M. (2010). La notion de diagnostic dans le cadre d'applications de la méthode verbo-tonale à l'apprentissage d'une Langue2/Langue Etrangère par des bilingues et à la rééducation de patients aphasiques. Glossa, (108), 53-68.

Bartolí, M. (2005). La pronunciación en la clase de lenguas extranjeras. Phonica, 1, 1-27. Recuperado de http://revistes.ub.edu/index.php/phonica/article/view/5565.

Blanche-Benveniste, C. (1991). Les études sur l'oral et le travail d'écriture de certains poètes contemporains. Langue française, (89), 52-71. doi: 10.3406/lfr.1991.5763.

Blanche-Benveniste, C. (1995). De quelques débats sur le rôle de la langue parlée dans les évolutions diachroniques. Langue française, (107). 25-35. doi: 10.3406/lfr.1995.5302.

Bolekia, J. (1989). La fonética francesa en la formación inicial de los alumnos-profesores. Aula, 2, 53-59. Recuperado de http://revistas.usal.es/index.php/02143402/article/view/3199/3223.

Buyssens, É. (1943). Les langages et le discours : essai de linguistique fonctionnelle dans le cadre de la sémiologie. Bruselas: Office de Publicité. 
Buyssens, É. (1980). Epistémologie de la phonématique. Bruselas: Éditions de l'Université de Bruxelles.

Capt-Artaud, M.-C. (1994). Petit traité de rhétorique saussurienne. Ginebra: Droz.

Chevalier, J.- C. (1997). Phonétique expérimentale et Cours pour étrangers en France à la fin du 19e siècle. En E. Hammar (Ed.), Phonétique et pratiques de prononciation. Documents pour l'histoire du français langue étrangère ou seconde (pp. 158-165). París, SIHFLES.

Constantin, E., y Saussure, F. (2005). Linguistique générale (Cours de M. le Professeur de Saussure) Semestre d'hiver 1910-1911. Cahiers Ferdinand de Saussure, (58), 83-289.

Detey, S. (2007). Transcription, translittération et didactique de l'oral en FLE au Japon: katakana, rômaji et orthographe française. Revue japonaise de didactique $d u$ français, 2(1), 19-36. doi: 10.24495/rjdf.2.1_19.

Giralt, M. (2006). El enfoque oral en la enseñanza del español como lengua extranjera: experiencia piloto de una propuesta didáctica. Phonica, 2, 1-26. Recuperado de http://revistes.ub.edu/index.php/phonica/article/view/5648.

Guimbretière, E. (2000). L'enseignement de la phonétique : état des lieux entre tradition et modernité. Mélanges CRAPEL, (25), 153-168. Recuperado de http://www.atilf.fr/IMG/pdf/melanges/11_guimbretiere.pdf.

Hjelmslev, L. (1971). Prolegómenos a una teoría del lenguaje (Trad. J. Díaz). Madrid: Gredos. (Traducción de Omkring Sprogteoriens Grundlæggelse, 1943, Copenhague: Københavns Universitet).

Li, J., Yin, Y., y Pu, Z. (2018). API et enseignement de la prononciation du français en Chine : médiation ou interférence ? $6^{e}$ Congrès Mondial de Linguistique Française CMLF 2018, Mons. doi: 10.1051/shsconf/20184607005.

Lightbown, P., y Spada, N. (2013). How Languages are Learned. Oxford: Oxford University Press.

Malmberg, B. (1991). Histoire de la Linguistique: de Sumer à Saussure. París: Presses Universitaires de France.

Meillet, A. (1925). La méthode comparative en linguistique historique. París: Honoré Champion.

Mejía, C. (1997). Unde exoriar ? Cahiers Ferdinand de Saussure, (50), 93-126.

Mejía, C. (1999). L'aposème, unité de parole. Cahiers Ferdinand de Saussure, (52), 237-252.

Mejía, C. (2000). La silhouette du mot. En D. Chiricò, N. Lamedica, y C. Stancati (Ed.), Parole come radici. Linguaggio e Filosofia tra storia e teoria (pp. 225-256). Rende: Brenner. Mejía, C. (2004). Propos sur l'homonymie. La recherche à l'heure de la psychanalyse. Rennes: Presses Universitaires de Rennes.

Mejía, C., y Marmolejo, S. (2010). Las prácticas de transferencia, laboratorio del habla. Entornos, (23), 93-103. Recuperado de https://journalusco.edu.co/index.php/entornos/article/view/1963. 
Mesa, A., y Mejía, C. (2011). La explicitación como estrategia pedagógica en los procesos de diseño. Revista Kepes, 8(7), 171-185. Recuperado de http://190.15.17.25/kepes/downloads/Revista7_10.pdf.

Passy, P. (1887). Les sons du français. Leur formation, leur combinaison, leur représentation. París: Firmin Didot.

Piferrer, F. (1847). El idioma francés puesto al alcance de todos, o Método natural para aprender el francés de un modo fácil y agradable sin cansar la memoria. Madrid: Despacho de Libros de la Calle Espoz y Mina.

Prieto, L. J. (1975a). L'écriture, code substitutif ? En Études de linguistique et sémiologie générales (pp. 85-93). Ginebra: Droz.

Prieto, L. J. (1975b). Pertinence et pratique : essai de sémiologie. París: Éditions de Minuit.

Prieto, L. J. (1983). Lingue grafiche e lingue foniche. En C. Vallini (Ed.), Scrittura e scritture: seminario interdisciplinare su teoria e prassi della scrittura (pp. 5-20). Nápoles: Istituto Universitario Orientale.

Prieto, L. J. (1991). Saggi di semiotica II. Sull'arte e sul soggetto. Parma: Pratiche Editric.

Richards, J. C., y Rodgers, T. S. (1986). The Audiolingual Method. En Approaches and Methods in Language Teaching (pp. 44-63). Cambridge: Cambridge University Press.

Saussure, F. (1879). Mémoire sur le système primitif des voyelles dans les langues indoeuropéennes. Leipzig: Teubner.

Saussure, F. (1968). Cours de linguistique générale. Édition critique par Rudolf Engler (Tomo 1). Wiesbaden: Otto Harrasowitz.

Saussure, F. (1974). Cours de linguistique générale. Édition critique par Rudolf Engler (Tomo 2). Wiesbaden: Otto Harrasowitz.

Saussure, F. (2019). Primer curso. Lingüística general de Ferdinand de Saussure, Louis Caille y Albert Riedlinger. Edición bilingüe de Claudia Mejía Quijano, Daniel Jaramillo Giraldo y Alexander Pérez Zapata. Medellín: Editorial Semsa.

Suso, J. (2002). Descriptions phonétiques et enseignement de la prononciation du français en Espagne au début du XXe siècle (jusqu'en 1936). Histoire de l'enseignement $d u$ français langue étrangère ou seconde dans le bassin méditerranéen, (28). Recuperado de http://journals.openedition.org/dhfles/2645.

Troubetzkoy, N. S. (1949). Principes de phonologie. París: Klincksieck.

\section{SOBRE LOS AUTORES}

\section{Claudia Lucia Mejía Quijano}

Doctora en letras (Lingüística general) de la Universidad de Ginebra. Profesora titular de la Universidad de Antioquia en el pregrado de Traducción inglés-francés-español, en la Maestría y el Doctorado de lingüística. Directora del grupo de investigación en semiología saussuriana - SEMSA. Sus áreas de investigación son la semiología y la lingüística generales.

Correo electrónico: lucia.mejia@udea.edu.co 
Orcid:_0000-0003-3366-480X.

\section{Daniel Jaramillo Giraldo}

Traductor inglés-francés-español y Magíster en Literatura de la Universidad de Antioquia. Profesor en la Universidad de Antioquia y la Universidad Católica de Oriente. Áreas de docencia e investigación: semiología, lingüística general, adquisición y enseñanza de lenguas extranjeras, literatura colombiana.

Correo electrónico: danielf.jaramillo@udea.edu.co

Orcid: 0000-0002-0637-7971.

\section{José Luis Betancur}

Se encuentra actualmente finalizando los estudios de pregrado en Licenciatura en Lenguas Extranjeras Inglés-francés en la Universidad de Antioquia. Sus intereses en la investigación son las lenguas extranjeras y la lingüística.

Correo electrónico: jluis.betancur@udea.edu.co

Orcid: 0000-0001-5765-7466. 